\title{
Incidencia de sobrepeso y obesidad según índice de masa corporal y perfil lipídico en estudiantes de Farmacia y Bioquímica de la Universidad Nacional Jorge Basadre Grohmann de Tacna, 2016
}

\author{
Incidence of overweight and obesity according to body mass index and lipid profile in Pharmacy and \\ Biochemistry students of the Jorge Basadre Grohmann National University of Tacna, 2016 \\ Incidência de sobrepeso e obesidade de acordo com índice de massa corporal e perfil lipídico em
} alunos de Farmácia e Bioquímica da Universidade Nacional Jorge Basadre Grohmann de Tacna, 2016

\author{
Orlando Agustín Rivera-Benavente ${ }^{1 a}$ \\ Mónica Karina Chipana-Flores ${ }^{1 a}$ \\ Gleny Betzabé Maquera-Flores ${ }^{16}$ \\ Adrian Alexis Romero-Guillen ${ }^{10}$ \\ José Luis Quispe-Pari ${ }^{10}$
}

\section{Resumen}

Objetivo: Determinar la incidencia de sobrepeso y obesidad según índice de masa corporal y perfil lipídico en estudiantes de Farmacia y Bioquímica de la Universidad Nacional Jorge Basadre Grohmann de Tacna. Material y métodos: Estudio observacional, descriptivo y correlacional. Se realizó toma de muestra de sangre en ayunas para análisis de laboratorio de perfíl lipídico y glicemia, y se evaluó peso y talla con cálculo de índice de masa corporal a una muestra no probabilíticas de 38 estudiantes de Farmacia y Bioquímica de la Facultad de Ciencias de la Salud. Los datos fueron recolectados en una ficha y procesados en el software estadístico SPSS versión 19, para el análisis se utilizó estadística descriptiva y para establecer la relación entre las variables se utilizó la prueba Chi cuadrado con un nivel de confianza de $95 \%$ y significancia asintótica de $<0,05$. Resultados: Los estudiantes presentaron edad de 18 a 26 años, con un promedio de 20,7 años, peso de $62,3 \mathrm{Kg}$ y talla de 1,61 m. Presentaron sobrepeso el $26,7 \%$ de mujeres y el $25 \%$ de varones. Según perfil lipídico: el 5,3\% presentaron colesterol total alto y riesgo moderado 18,4 \%, todos HDL colesterol normal, 26,3 \% LDL colesterol alto, 5,3 \% presentaron valores altos de trigliceridos y $31,6 \%$ hipoglicemia leve. Todos los varones $n$ presentaron valores normales de colesterol total, HDL y LDL; y todas las mujeres presentaron triglicéridos normales. En cuanto a actividad física, el $26,3 \%$ realiza poca actividad física y el $18,4 \%$ no realiza ninguna actividad física. No se encontró diferencias estadísticamente significativas entre sexo femenino y masculino ( $p>0,05)$. Conclusión: La incidencia de sobrepeso y obesidad es más frecuente en mujeres, y en su perfil lipídico presentan valores altos de colesterol total, LDL colesterol y trigliceridos.

Palabras clave: sobrepeso, obesidad, índice de masa corporal, colesterol, triglicéridos

\footnotetext{
${ }^{1}$ Universidad Nacional Jorge Basadre Grohmann, Escuela Profesional de Farmacia y Bioquimica. Tacna, Perú ${ }^{a} Q u i ́ m i c o$ farmacéutico

${ }^{\mathrm{b}}$ Servidor administrativo profesional

'Estudiantes de Farmacia y Bioquímica
} 


\begin{abstract}
Objective: To determine the incidence of overweight and obesity according to body mass index and lipid profile in Pharmacy and Biochemistry students of the Jorge Basadre Grohmann National University, Tacna. Material and methods: Observational, descriptive and correlational study. Fasting blood sample was taken for laboratory analysis of lipid perfyl and glycemia, and weight and height were evaluated with calculation of body mass index to a non-probabilitic sample of 38 students of Pharmacy and Biochemistry of the Health Sciences Faculty. The data were collected on a data sheet and processed in the statistical software SPSS v19. Descriptive statistics were used for the analysis. The square Chi test was used to establish the relationship between the variables, with a confidence level of $95 \%$ and asymptotic significance of $<0.05$. Results: The students were 18 to 26 year-old, with an average of 20.7 , weight of $62.3 \mathrm{Kg}$ and height of $1.61 \mathrm{~m} .26 .7 \%$ of women and $25.0 \%$ of men were overweight. According to lipid profile: $5.3 \%$ had high total cholesterol and moderate risk $18.4 \%$, all normal HDL cholesterol, $26.3 \%$ LDL high cholesterol, $5.3 \%$ had high triglyceride values and $31.6 \%$ mild hypoglycemia. All men had normal values of total cholesterol, HDL and LDL; and all women had normal triglycerides. About physical activity, $26.3 \%$ do little physical activity and $18.4 \%$ do not do any physical activity. No statistically significant differences were found between female and male gender $(p>0.05)$. Conclusion: The incidence of overweight and obesity is more frequent in women, and in their lipid profile have high levels of total cholesterol, LDL cholesterol and triglycerides.
\end{abstract}

Keywords: overweight, obesity, body mass index, cholesterol, triglycerides

\title{
Resumo
}

Objetivo: Determinar a incidência de sobrepeso e obesidade segundo índice de massa corporal e perfil lipídico em estudantes de Farmácia e Bioquímica da Universidade Nacional Jorge Basadre Grohmann de Tacna. Material e métodos: Estudo observacional, descritivo e correlativo. Foi realizada amostragem de sangue em jejum para análise laboratorial de perfilagem lipídica e glicemia, e foi avaliado peso e tamanho com cálculo de índice de massa corporal a uma amostra não probabilítica de 38 estudantes de Farmácia e Bioquímica da Faculdade de Ciências da Saúde. Os dados foram coletados em uma ficha e processados no software estatístico SPSS versão 19, para a análise utilizou-se estatística descritiva e para estabelecer a relação entre as variáveis utilizou-se o teste Chi quadrado com um nível de confiança de $95 \%$ e significância assintótica de <0,05. Resultados: Os estudantes apresentaram idade de 18 a 26 anos, com uma média de 20,7 anos, peso de $62,3 \mathrm{Kg}$ e tamanho de $1,61 \mathrm{~m}$. Apresentaram sobrepeso $26,7 \%$ de mulheres e $25 \%$ de homens. Perfil lipídico: 5,3\% apresentaram colesterol total alto e risco moderado $18,4 \%$, todos HDL colesterol normal, 26,3 \% LDL colesterol alto, 5,3\% apresentaram valores elevados de trigliceridos e 31,6\% hipoglicemia leve. Dos homens, todos apresentaram valores normais de colesterol total, HDL e LDL; e das mulheres, todas apresentaram triglicéridos normais. No que se refere à atividade física, $26,3 \%$ têm pouca actividade física e 18,4 \% não exercem qualquer atividade física. Não foram encontradas diferenças estatisticamente significativas entre o sexo feminino e masculino $(p>0,05)$ Conclusão: $A$ incidência de sobrepeso e obesidade é mais freqüente em mulheres, e em seu perfil lipídico apresentam valores elevados de colesterol total, LDL colesterol e trigliceridos.

Palavras-chave: sobrepeso, obesidade, índice de massa corporal, colesterol, triglicéridos

\section{Introducción}

La obesidad y el sobrepeso se han incrementado en forma alarmante en las últimas décadas. Considerándose una epidemia global y es la enfermedad no transmisible más prevalente en el mundo que afecta desde edades más tempranas; ya que, según datos de 2014, más de 1900 millones de adultos de 18 o más años tenían sobrepeso, de los cuales, más de 600 millones eran obesos. Y la mayoría de la población mundial vive en 
países donde el sobrepeso y la obesidad cobran más vidas de personas. ${ }^{1}$

Los adolescentes han abandonado paulatinamente la actividad física, el deporte y los juegos al aire libre, permaneciendo más tiempo frente a las pantallas: celulares, computadoras, televisión. ${ }^{2}$ Esto resulta en un estilo de vida sedentario y poco saludable, que constituye una de las principales causas de la obesidad y el sobrepeso. Si se mantiene el sobrepeso a lo largo del tiempo, pueden aparecer enfermedades como diabetes, hipertensión, problemas osteo-articulares, cardiovasculares y otras.

En poblaciones con un alto grado de adiposidad el exceso de grasa corporal (adiposidad) esta altamente correlacionado con el peso corporal. Por esta razón, el índice de masa corporal (IMC) es una medición válida y conveniente. Para el presente estudio se utilizó el IMC como indicador para determinar el grado de sobrepeso y obesidad, el cual se calcula al dividir el peso en kilogramos sobre el cuadrado de la talla en metros $\left(\mathrm{IMC}=\mathrm{Kg} . / \mathrm{m}^{2}\right)$. Es una indicación simple la relación entre el peso y la talla al cuadrado que se utiliza frecuentemente para identificar el sobrepeso y la obesidad en los adultos, tanto a nivel individual como poblacional. La Organización Mundial de la Salud (OMS) define el sobrepeso como un IMC igual o superior a 25 , y la obesidad como un IMC igual o superior a $30 .^{3}$

La actividad física, definida como cualquier movimiento corporal producido por los músculos esqueléticos que resulte en gasto calórico, desempeña un papel importante en la prevención de las enfermedades crónicas no transmisibles y disminuye el riesgo de obesidad, actuando en la regulación del balance energético y preservando o manteniendo la masa magra en detrimento de la masa grasa. ${ }^{4}$

Es necesario señalar que la monitorización del estado nutricional requiere el empleo de varios test de laboratorios usados conjuntamente con cada uno de los datos derivados del método antropométrico y también de la observación de signos clínicos y síntomas de deficiencias nutricionales. ${ }^{5}$ Comparados con otros métodos de valoración, los test de laboratorio tienen la ventaja de su carácter objetivo y cuantitativo, y los de mayor importancia para identificar la incidencia de obesidad y sobrepeso asociado con enfermedades crónicas es la valoración de los exámenes de perfil lipídico (colesterol total, HDL colesterol, LDL colesterol triglicéridos y glucosa).

En los últimos años, en Tacna se han experimentado transformaciones socioeconómicas y demográficas, caracterizadas por una rápida urbanización y globalización, que han modificado profundamente el modo de vivir de sus habitantes. Esto ha traído como consecuencia el incremento en el consumo de alimentos altamente calóricos, conjuntamente con un aumento significativo de inactividad física y obesidad en todos los grupos etarios.

Tacna no está ajena a esta situación, de acuerdo al Monitoreo Nacional de Indicadores Nutricionales INS/CENAN (2014), Ia prevalencia de obesidad fue de $1,9 \%$. Esto indica que la obesidad va en aumento tanto a nivel regional como nacional. ${ }^{6}$ Razón por la cual se realizó la presente investigación, con la finalidad de determinar la incidencia de sobrepeso y obesidad, según índice de masa corporal y perfil lipídico en estudiantes de Farmacia y Bioquímica de la Universidad Nacional Jorge Basadre Grohmann de Tacna.

\section{Material y métodos}

Estudio observacional, descriptivo y correlacional, con muestra no probabilistica de 38 estudiantes de la Escuela Profesional de Farmacia y Bioquímica de la Facultad de Ciencias de la Salud de la Universidad Nacional Jorge Basadre Grohmann de Tacna.

Se realizó entrevista, toma de muestra de sangre en ayunas con análisis de laboratorio de perfíl lipídico y evalución de peso y talla con el cálculo de índice de masa corporal a los estudiantes de Farmacia y Bioquimica. Para el procesamiento de datos se utilizó el sofware SPSS versión 19, para el análisis estadístico descriptivo y para establecer la relación entre las variables se utilizó la prueba estadística del 
Chi cuadrado con un nivel de confianza de $95 \%$ y significancia asintótica de <0,05.

\section{Resultados}

En el estudio se observa que del total de 38 estudiantes de Farmacia y Bioquímica, la edad mínima fue de 18 años, la máxima de 26 años, y el promedio de 21 años. El peso mínimo fue de 45 kilogramos y el máximo de 106 kilogramos, con un peso promedio de $62 \mathrm{~kg}$. La talla mínima fue de 1,44 metros y la máxima de 1,85 metros, con un promedio de 1,62 metros (Tabla 1). En relación al perfil lipídico: en colesterol total se observa que el 23,3\% (7 estudiantes del sexo femenino) presentan valores de riesgo moderado, el $6,7 \%$ (2 estudiantes del sexo femenino) presentan valores de riesgo alto (Tabla 2). En cuanto a valores de colesterol HDL coleterol de los estudiantes, se encuentran dentro de los valores normales (Tabla 3). En relación a valores de colesterol LDL colesterol se observa que el 33,3\% (10 estudiantes del sexo femenino) presentan valores de riesgo alto (Tabla 4). Se evidencia en el estudio, que el 5,3\% (2 estudiantes del sexo masculino) presentan valores altos de trigliceridos (Tabla 5). Del total de 38 estudiantes de Farmacia y Bioquímica, se observa que el 31,6 \% (2 estudiantes del sexo masculino y 10 estudiantes del sexo femenino) presentan valores de hipoglicemia leve (Tabla 6).

Tabla 1

Estadísticos descriptivos de las edades, peso y talla

\begin{tabular}{lccccc}
\hline & $\mathrm{N}$ & Mínimo & Máximo & Media & Desv. típ. \\
\hline Edad (años) & 38 & 18 & 26 & 20,7368 & 1,92683 \\
Peso $(\mathrm{kg})$ & 38 & 45 & 106 & 62,2895 & 11,41575 \\
Talla $(\mathrm{m})$ & 38 & 1,44 & 1,85 & 1,6163 & 0,08756 \\
\hline
\end{tabular}

\section{Tabla 2}

Resultados de los valores de colesterol total en los estudiantes de Farmacia y Bioquímica- FACSUNJBG, según sexo

\begin{tabular}{lllccc}
\hline & & \multicolumn{3}{c}{ Sexo } \\
& & & Masculino & Femenino & Total \\
\hline $\begin{array}{l}\text { Colesterol } \\
\text { total }\end{array}$ & Normal "<200mg/dL" & Número & 8 & 21 & 29 \\
& & $\%$ & 100 & 70 & 76,3 \\
& & & & & 7 \\
& Riesgo Moderado & Número & 0 & 23,3 & 18,4 \\
& "200 - 240 mg/dL" & $\%$ & 0 & 2 & 2 \\
& Riesgo Alto "> 240 mg/dL" & Número & 0 & 6,7 & 5,3 \\
& & $\%$ & 0 & 30 & 38 \\
& & Número & 8 & 100 & 100 \\
\hline
\end{tabular}




\section{Tabla 3}

Resultados de los valores de HDL-colesterol en los estudiantes de la "E.P. de Farmacia y BioquímicaFACS-UNJBG", según sexo

\begin{tabular}{lllcrc}
\hline & & & Sexo & \\
& & Masculino & Femenino & Total \\
\hline \multirow{2}{*}{ HDL Normal "> 25 mg/dL" } & Número & 8 & 30 & 38 \\
& $\%$ & 100 & 100 & 100 \\
\multirow{3}{*}{ Total } & Número & 8 & 30 & 38 \\
& $\%$ & 100 & 100 & 100 \\
\hline
\end{tabular}

Tabla 4

Resultados de los valores de LDL-colesterol en los estudiantes de Farmacia y Bioquímica-FACSUNJBG, según sexo

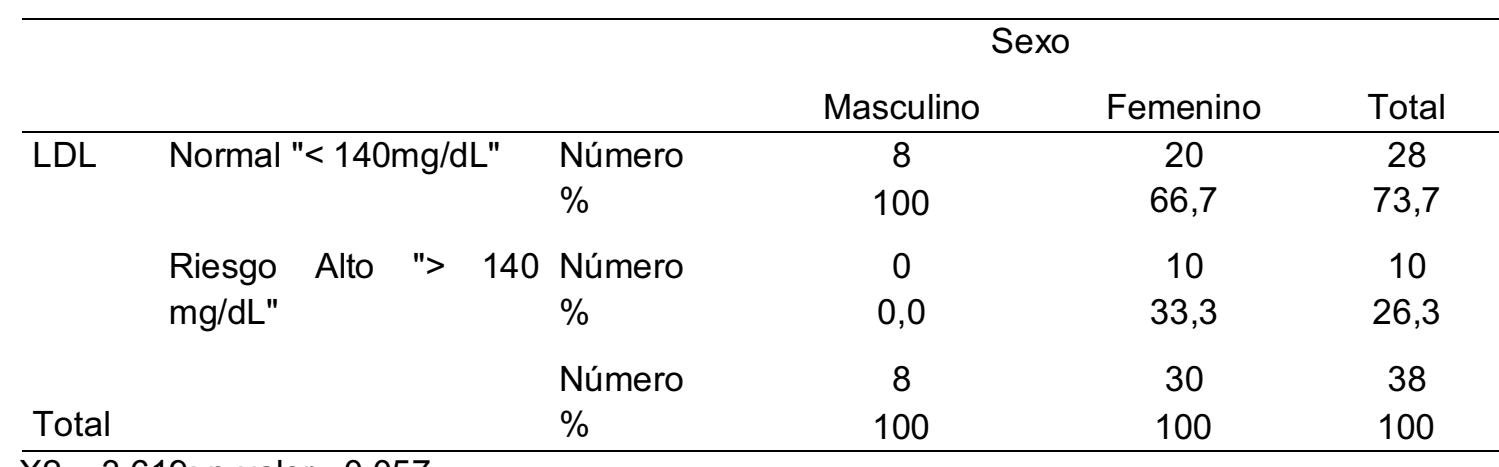

$\mathrm{X} 2=3,619 ; \mathrm{p}$-valor $=0,057$

\section{Tabla 5}

Resultados de los valores de triglicéridos en los estudiantes de Farmacia y Bioquímica- FACSUNJBG, según sexo

\begin{tabular}{|c|c|c|c|c|c|}
\hline & & & \multicolumn{2}{|c|}{ Sexo } & \multirow[b]{2}{*}{ Total } \\
\hline & & & Masculino & Femenino & \\
\hline \multirow[t]{4}{*}{ Triglicéridos } & Normal "<165mg/dL" & Número & 6 & 30 & 36 \\
\hline & & $\%$ & 75 & 100 & 94,7 \\
\hline & Riesgo & Número & 2 & 0 & 2 \\
\hline & mg/dL" & $\%$ & 25 & 0 & 5,3 \\
\hline \multirow[t]{2}{*}{ Total } & & Número & 8 & 30 & 38 \\
\hline & & $\%$ & 100 & 100 & 100 \\
\hline
\end{tabular}

$\mathrm{X} 2=7,917 ; \mathrm{p}$-valor $=0,005$ 


\section{Tabla 6}

Resultados con los valores de glucosa en los estudiantes de Farmacia y Bioquímica-FACS-UNJBG, según sexo

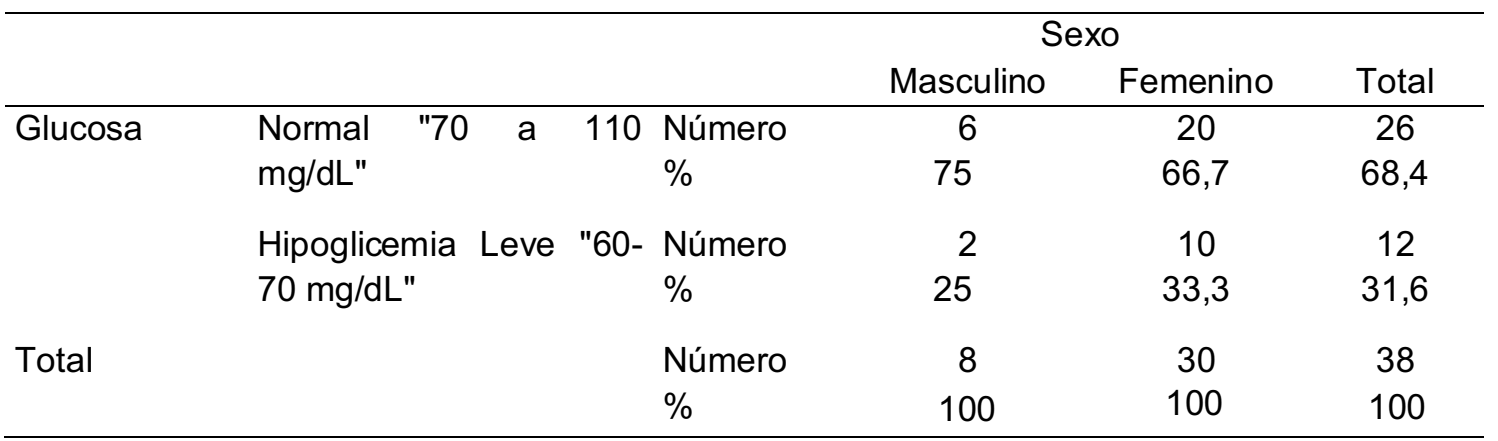

$\mathrm{X} 2=0,203 ; \mathrm{p}$-valor $=0,652$

\section{Discusión}

La obesidad y sobrepeso constituye un indicador que permite conocer como es la incidencia de sobrepeso y obesidad según índice de masa corporal y perfil lipídico en estudiantes de la E.P. de Farmacia y BioquímicaFACS de la Universidad Nacional Jorge Basadre Grohmann de Tacna, 2016.

En el total de 38 estudiantes de la E.P. de Farmacia y Bioquímica encuestados, según grupo etáreo, se puede apreciar que el promedio de edad es de 21 años, en alumnos de Farmacia y Bioquímica para, Peña, S. ${ }^{7}$ Ecuador en el 2014, en su estudio, "Factores asociados a la obesidad abdominal en estudiantes de Medicina de la Universidad Católica cuenca, 2014" encontró resultados similares según grupo etario menciona que la edad promedio fue de 21,57 años, según sexo, el $78,9 \%$ predomina el sexo femenino y el $21,1 \%$ son de sexo masculino, que corresponde con lo descrito por Reyes, et al., ${ }^{8}$ Guatemala en 2013 , en su estudio titulado "Prevalencia de factores de riesgo modificables en enfermedades crónicas no transmisibles", se encontró resultados similares en el porcentaje de sexo femeninos $52 \%$.

En cuanto al peso, se puede apreciar que el promedio es de $62 \mathrm{~kg}$, los resultados de la investigación no concuerdan con lo descrito por Rangel, et al., ${ }^{9}$ Colombia en el 2013, en su estudio "Sobrepeso y obesidad en estudiantes universitarios colombianos y su asociación con la actividad física de la Universidad Privada de
Bucaramanga, Colombia en el año 2013", con el promedio de peso de $26,47 \%$.

Reyes, et al., ${ }^{8}$ Guatemala en 2013, realizó un estudio sobre "Prevalencia de factores de riesgo modificables en enfermedades crónicas no transmisibles" dio como resultado con un $23 \%$ con sobrepeso del sexo famenino. Coinside con nuestro estudio según índice de masa corporal (IMC) con el $26,7 \%$ del sexo femenino y $25,0 \%$ del sexo masculino se encuentran con sobrepeso. Se determinó un caso de desnutrición con sexo femenino que corresponde al 3,3\%. Que corresponde con lo descrito por Gallardo y Buen, ${ }^{10}$ México en el 2010, realizaron un estudio sobre "Mala nutrición en estudiantes universitarios de la Escuela de Dietética y Nutrición del ISSSTE" dio como resultado $15,1 \%$ con sobrepeso y $26,4 \%$ con desnutrición del sexo femenino.

Pinedo y Vargas, ${ }^{11}$ Perú en el 2009 , en su estudio "Prevalencia de sobrepeso y obesidad en pacientes atendidos en la consulta externa del Hospital III - Iquitos- Essalud - 2009", que dio como resultado el colesterol elevado en pacientes con sobrepeso (91,7\%), asimismo, resultaron con Colesterol HDL y LDL elevado un $(92,3 \%)$ en pacientes con sobrepeso, en relación a los análisis de triglicéridos fue elevado en pacientes con sobrepeso $(89,7 \%)$. En la presente investigación, se encuentra similar información, según colesterol total, de un total de 38 estudiantes de la E.P. de Farmacia y Bioquímica, el 6,7 \% presenta colesterol total 
alto, LDL colesterol se puede observar que el $33,3 \%$ tiene LDL colesterol alto, y el 5,3\% muestran valores altos de triglicéridos.

\section{Referencias}

1. Organización Mundial de la Salud (OMS). Centro de prensa. Nota descriptive. Suiza. [Internet]. [citado 02 de junio 2016]. D i s p o n i b I e e $n$ : http://www.who.int/mediacentre/factsheets/ fs $385 /$ es/

2. Grunbaum, S. Sobrepeso y obesidad en los adolescentes. Asociación española primera en salud. España [Internet]. 2015 [citado 04 de junio 2016]. Disponible en: http://www.asesp.com.uy/uc_613_1.html

3. Organización Mundial de la Salud. Use and interpretation of anthopometric indicators of nutritional status.Bulletin of the World Health Organization Bulletin de I' Organisation mondiale de la Santé. Grupo de Trabajo de la OMS. Suiza. [Internet]. 2006 [citado 04 de junio 2016]; 64:929-941.

4. Seclen-Palacin J, Jacoby E. Factores sociodemográficos y ambientales asociados con la actividad física deportiva en la población urbana del Perú. Rev. Panamá. Salud Pública; [Internet]. 2003 [citado 04 de junio 2016]; 14(4):255-64.

5. Quesada A. Diagnóstico de Laboratorio. Principales pruebas de Bioquímica Clínica y de Laboratorio. Primera Edición. Litografía e Imprenta Lehmann. Tibás. San José, Costa Rica. [Internet]. 2005 [citado 04 de junio 2016].

6. Callomamani L. Relación entre el sobrepeso-obesidad y la actividad física en escolares de 9 a 11 años de la I.E. Mariscal Cáceres Tacna - 2012. [Tesis para optar el título profesional de licenciada en enfermería]. Tacna. Universidad Nacional Jorge Basadre Grohmann- Tacna. 2013 [citado 07 de junio 2016]; Disponible en: http://repositorio.unjbg.edu.pe/bitstream/h andle/UNJBG/397/TG0252.pdf?sequence $=1$ \&isAllowed $=\mathrm{y}$

7. Peña S. Factores asociados a la obesidad abdominal en estudiantes de medicina de la Universidad Católica Cuenca, 2014. [Tesis de Especialidad en Medicina Interna]. [Internet]. 2014 [citado 08 de junio 2016]; $\mathrm{D}$ i s p o n i b le $\mathrm{n}$ : http://dspace.ucuenca.edu.ec/bitstream/12 3456789/21271/1/TESIS.pdf.

8. Reyes S. Prevalencia de factores de riesgo modificables en enfermedades crónicas no transmisibles, 2012. [Tesis de Medicina]. Universidad de San Carlos de Guatemala. 2013 [citado 08 de junio 2016]. Disponible http://biblioteca.usac.edu.gt/tesis/05/05_89 07.pdf.

9. Rangel L, Rojas L, Gamboa E. Sobrepeso y obesidad en estudiantes universitarios colombianos y su asociación con la actividad física. [Tesis Doctoral], 2013. Colombia, [citado 08 de junio 2016]. D i s p o n i ble e n : http://www.aulamedica.es/nh/pdf/7757.pdf.

10. Gallardo I, Buen L. Mala nutrición en estudiantes universitarios de la Escuela de Dietética y Nutrición del ISSSTE. 2010 D i s p n i b le e n : https://www.uv.mx/rm/num_anteriores/rev medica_vol11_num1/artículos/mala.pdf.

11. Pinedo $P$, Vargas M. Prevalencia de sobrepeso y obesidad en pacientes atendidos en la consulta externa del hospital III -lquitos- Essalud - 2009" [Tesis para optar el título de Químico Farmacéutico]. Perú, Universidad Nacional de la Amazonia Peruana. 2009. [citado 08 de junio 2016]. Disponible en: http://repositorio.unapiquitos.edu.pe/bitstre am/handle/UNAP/3675/Priscila_Tesis_Titul o_2009.pdf?sequence $=1$ \&isAllowed $=\bar{y}$

\section{Correspondencia:}

oriverab@unjbg.edu.pe
Fecha de recepción: 20/07/2020

Fecha de aceptación: 13/01/2021 